\title{
A Study on Flexible Vibratory Feeding System Based on Smart Camera
}

\author{
Liang Hana, Huimin Lib \\ School of Mechanical Engineering, Southeast University, Jiangning \\ District, Nanjing 211189, China \\ amelhan@seu.edu.cn,blihuimin_2@126.com
}

\begin{abstract}
Combining machine vision technology and vibratory feeder equipment, this paper designs a set of flexible vibratory feeding system based on smart camera. This system collects an image of the workpiece by the smart camera Vision MINI and then defines the correct main features of the workpiece such as posture, shape, and length with AutoVision software. These features are taken as standards to be compared with the corresponding features of the moving workpieces at the outlet of the vibratory feeder. Once the different features of a workpiece are identified, the system controller sends a signal to make the workpiece back into the bowl. If the workpiece is changed, it is only needed to change its feature definition in the software rather than change the mechanical structure of the vibratory feeder. Thus, it improves the flexibility of the vibratory feeding system and reduces the cost greatly. This study is significant to research an automatic manufacturing system which has ability to respond rapidly to changing demands in today's complex markets.
\end{abstract}

Keywords: Vibratory feeding; AutoVision software; Smart Camera; Features

\section{Introduction}

Vibratory feeding system is one of the most important parts of automatic assembly technology. It directly affects production efficiency and quality of assembly. According to the survey, more than $50 \%$ of total manufacturing cost is for assembly and more than $30 \%$ of assembly cost is for feeding workpiece. Vibratory feeder is the most widely used among the continuous feeding devices and adopted in food processing, pharmaceutical industry, electronic components assembly and other manufacturing fields [1]. It has advantages of simple structure, low consumption of energy, reliable working condition and adjustable feeding rate [2]. In the automatic assembling production line, functions of vibratory feeder are mainly shown in three aspects: storage of workpieces, feeding workpieces and orientation. The function of automatic orientation is the most important function among them. In order to make sure the workpiece is in the correct posture, an appropriate orientating mechanism is used when the workpiece is moving. The traditional vibratory feeder orients workpieces by 
adding orientating mechanisms like baffle, slope, groove, gap, track and footstep according to the features of workpieces. Traditional vibratory feeder lacks flexibility. If the shape or size of the workpiece changes, the orientating mechanism usually has to be redesigned. Sometimes, the shape or size changes a little while the new design and bowl processing maybe take several months, which obviously can not meet the rapidly changing market [3].

\section{Overall Design of Flexible Vibratory Feeding System}

The structure of flexible vibratory feeding system is shown in Fig.1. It consists of electromagnetically driven vibratory bowl feeder, smart camera, control circuit, electromagnetic valve and air nozzle. The vibratory bowl feeder makes the workpiece move upward along the track by its vibration. The smart camera collects images of the workpiece in real-time at the end of outlet of vibratory feeder. It compares the image with the standard image features. The air nozzle is fixed on the side of outlet of vibratory feeder, connected with a compressed air source through the electromagnetic valve. The electromagnetic valve is controlled by the control circuit. If the image that the smart camera acquires is not consistent with the standard image, the smart camera sends a signal to the control circuit, the controller open the electromagnetic valve. The workpiece is blown back into the bowl of vibratory feeder.

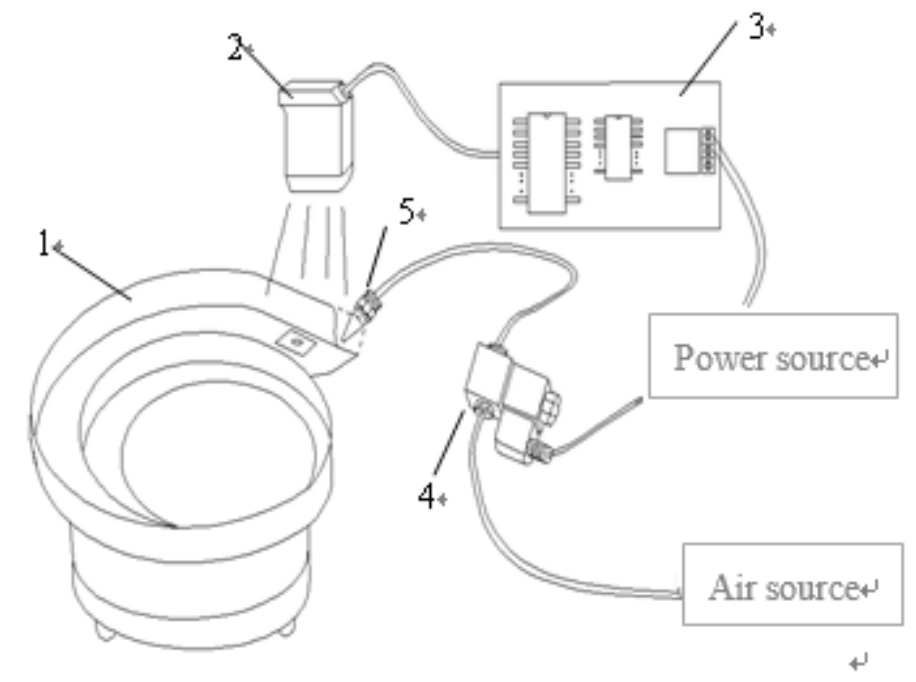

1. Vibratory bowl feeder; 2.Smart camera; 3.The control circuit board;

4.Electromagnetic valve; 5 . Air nozzle

Fig.1 The overall design of flexible vibratory feeding system based on smart camera

It is not necessary to add any orientating mechanism to the vibratory bowl feeder near its outlet. Only a small region over the outlet is needed for image acquisition. In order to make the workpiece distinguished from its background 
easily by the smart camera, the track of the vibratory feeder can be changed to black. The smart camera used in this study is Vision MINI series developed by Microscan. It integrates lens, a light source, the microcontroller and its digital I/O interface. The electromagnetic valve is two-position two-port valve. Air source is an air compressor. The electric control schematic diagram is shown in Fig.2.

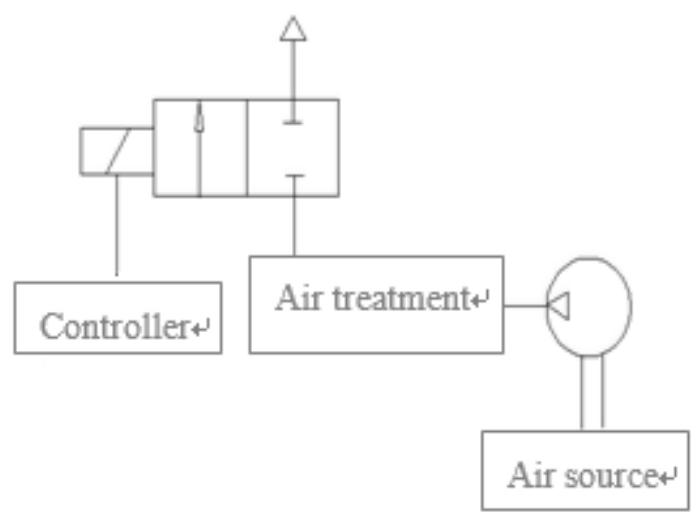

Fig.2 The electric control schematic diagram

The air nozzle is fixed on the outlet of vibratory feeder with a fixed block, as shown in Fig.3. The air nozzle and the fixed block are connected with small interference fit. This method is beneficial to the alignment of air nozzle's position.

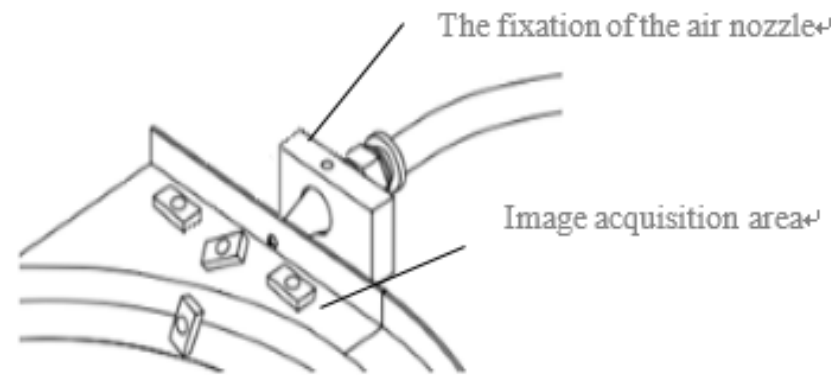

Fig.3 The outlet of vibratory feeder

\section{Introduction of Flexible Vibratory Feeding System Software}

AutoVision software is a new generation of machine vision software that is researched and developed by Microscan. This software provides user with an intuitional software interface, as shown in Fig.4. It integrates some powerful visual tool libraries such as location, counting, the one-dimensional code recognition, two-dimensional code recognition, distance detection, feature identification, feature comparison, etc. Through the use of these visual tool libraries, user can easily define corresponding features of the workpiece. In real- 
time detection, the software can quickly identify the features of a target image, compare them with the standard feature definition, and determine whether they are consistent or not. User can set the input/output signal of the camera to meet various logic controls. This software replaces traditional digital processing method that requires user to write some programs, including image acquisition, image processing and signal output. It is highly integrated to provide user with a simple platform. The user who does not understand fundamental algorithms of machine vision can be also qualified for visual detection, error prevention and automatic identification tasks. So this software gains the favors of many manufacturing engineers quickly.

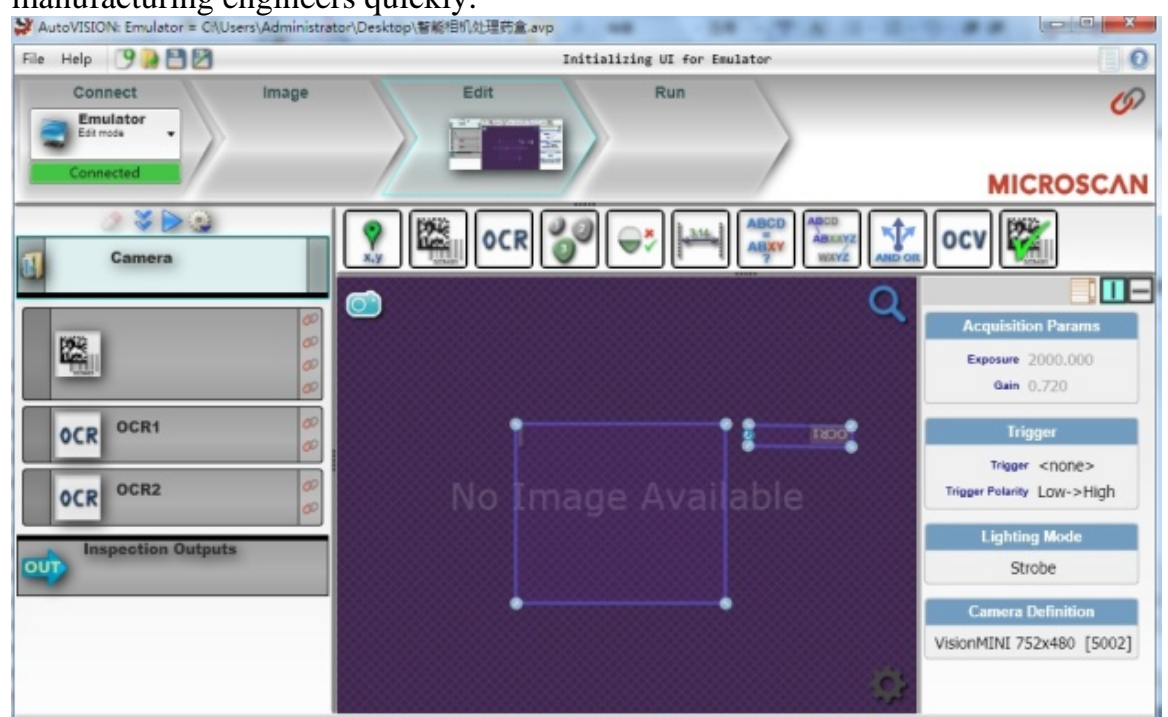

Fig.4 The software interface of AutoVision

\section{Experimental Results}

This study takes a kind of workpieces for an example to carry out the experiment. Firstly, some important features are defined in AutoVision software by using location, presence and measure tools. Location tool detects the center position of the workpiece in a specified area. Presence tool can distinguish whether this workpiece exists in the specified area or not. Measure tool can measure the width of the workpiece. Standard features of the workpiece are shown in Fig.5 and Fig.6. The feature information is stored in the smart camera. Secondly, set the output signal according to the logic required in this study. Finally, start the vibratory feeder. The smart camera collects images and determines whether they are the same with the standard or not, then outputs the signal to the external circuit. If the images are the same with the standard, the workpiece is transported out. Otherwise, the smart camera sends a signal to make the workpiece back into the bowl. Massive statistic data from those experiments shows that the efficiency of vibratory feeder is about 3-5 pieces per second, and the accuracy rate can be as high as $90 \%$. 

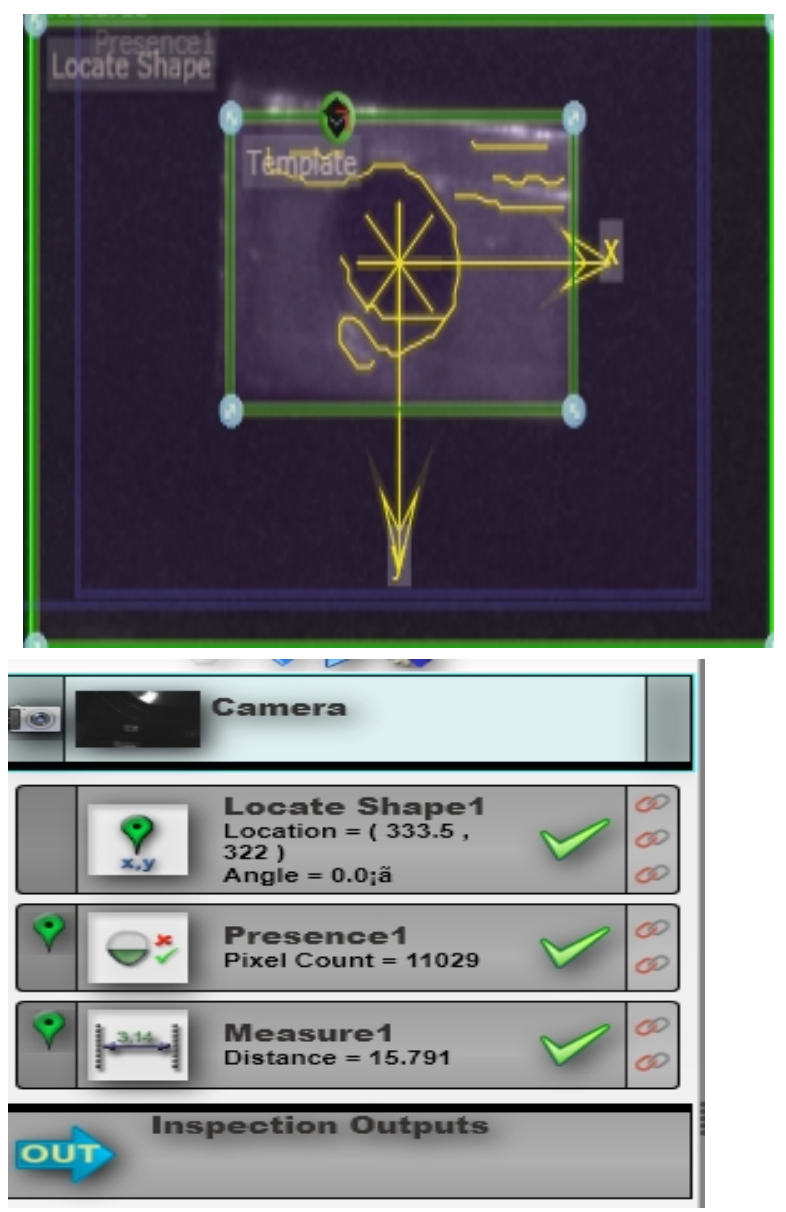

Fig 5.Schematic diagram of the feature definition

Fig 6.Inspection outputs of the recognition

\section{Conclusion}

Flexible vibratory feeding system based on smart camera overcomes some disadvantages caused by orientating mechanism such as low flexibility, high processing cost, low efficiency, etc. It not only can meet various detective requirements but also needs a short development cycle. Using smart camera to collect digital image, without contacting the workpiece, avoids causing any harm to the workpiece and meanwhile ensures the reliability of the whole system. This system can work in some harsh environment, such as high temperature workplace, toxic workplace and so on. This study plays an important role in researching the automatic manufacturing system that can respond rapidly to the changing market. 


\section{Acknowledgement}

This work is supported by NSFC, project No.:51275087.

\section{Reference}

[1] L Han, W L Xu. Status and Outlook of the Vibratory Feeder. Equipment for Electronic Products Manufacturing, 1999(1)

[2]X D Ding. Structure Design of the Vibratory Feeder Equipment. ElectroMechanical Engineering, 2007(06)

[3] L Han, C B Li, G P Hu. A Study on the Vision-Based Flexible Vibratory Feeding System. Advanced Materials Research, 2011, 279: 434-439

[4] W Pan. The Research About the Recognition and Positioning of the Workpiece Based on Machine Vision. [D] Bei Jing: Beijing University of chemical technology.2012 .13-19

[5] X F Li .Research of Auto Parts Defects Detection Technology Based on Machine Vision. [D] Wu Han: Wuhan University of Technology.2012.10-11

[6] Q Wang. Study on the Parts' Pose in Electromagnetic Vibrating Feeding. [D] He Bei: Yanshan University.2011.8-10

[7] L Zhou, G Jin. Control Technique of Feeding at a Constant Distance. [J] Mechanical Engineering and Automation. 2010(04)

[8] Daniel, Boejlk Smat. Design for Flexible Feeding .Machine Desion.1994

[9] A .H. Redford, G. Boothroyd. Vibratory feeding. Proceedings of the Institution of Mechanical Engineers. 1986

[10] G. P. Maul, N. I. Jaksic. Sensor-Based Solution to Contiguous and Overlapping Parts in Vibratory Bowl Feeders. Journal of Manufacturing Systems. 1994

[11] Ou - Yang, C, Maul G P.A Computer Analysis of Orientation Devices for Vibratory Bowl Feeders. International Journal of Production Research. 1993 